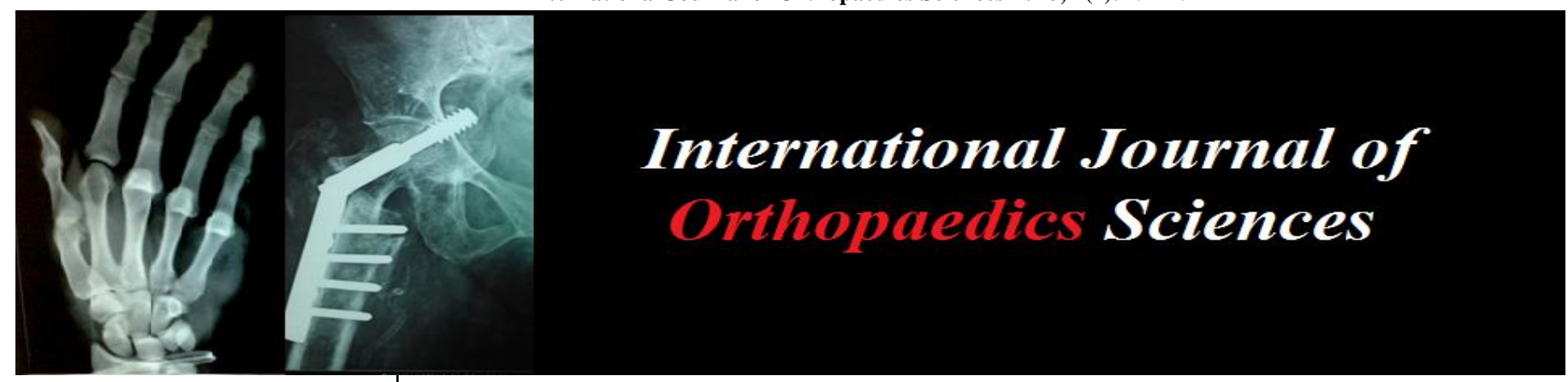

ISSN: $2395-1958$

IJOS 2018; 4(1): 792-794

(C) 2018 IJOS

www.orthopaper.com

Received: 03-11-2017

Accepted: 04-12-2017

Dr. Manvendra Singh Rawat Junior Resident Orthopaedics, Himalayan Institute of Medical Sciences, Uttarakhand, India

Dr. Anil Juyal

Professor Orthopaedics,

Professor Orthopaedics

Himalayan Institute of Medical

Sciences, Dehradun,

Uttarakhand, India

Dr. Atul Agrawal

Professor Orthopaedics

Himalayan Institute of Medical

Sciences, Dehradun,

Uttarakhand, India

\section{Evaluation of the role of intra-articular Steroid injection in frozen shoulder}

\author{
Dr. Manvendra Singh Rawat, Dr. Anil Juyal and Dr. Atul Agrawal
}

DOI: https://doi.org/10.22271/ortho.2018.v4.i11.113

\begin{abstract}
Introduction: Frozen shoulder is an idiopathic and progressive disease, which is identified by pain and decreased range of motion (ROM) of the shoulder and shoulder joint capsule fibrosis. It typically occurs in the fifth and sixth decades of life, thus affecting individuals of working age. Various author have quoted incidence of $2 \%-5 \%$ in general population. Different modalities of treatments like physical therapy, exercises, articular stretching and pulley therapy, physical therapy with medication. were used but, the combination of physical therapy and medication have shown better results. We evaluated the role of intra articular corticosteroid injection as it decreases inflammation which leads to reduction in capsular fibrosis. This allows enhancement of joint motion and reduces the functional recovery time. In this study we included 32 patients with primary diagnosis of frozen shoulder with duration of pain more than or equal to two months. All the patients fulfilling the inclusion criteria were mobilized under short General Anaesthesia followed by administration of intraarticular steroid $(2 \mathrm{ml}, 80 \mathrm{mg})$. Constant shoulder score (CSS) \& Visual Analogue Scale (VAS) were used as study tool for scoring. All the patients were subsequently reviewed and evaluated at 2, 6 and 12 weeks on the basis of VAS and CSS score which was found to be highly significant.
\end{abstract}

Keywords: Frozen shoulder, Periarthritis, adhesive capsulitis

\section{Introduction}

Frozen shoulder is an idiopathic and progressive disease, which is identified by pain and decreased range of motion (ROM) of the shoulder and shoulder joint capsule fibrosis (1).It is an extremely painful and debilitating condition leading to stiffness and disability. It typically occurs in the fifth and sixth decades of life, thus affecting individuals of working age. Frozen shoulder can be either primary (idiopathic) or secondary. Secondary frozen shoulder is defined as that associated with trauma; rotator cuff disease and impingement; cardiovascular disease; hemiparesis; or diabetes ${ }^{[2]}$.

This condition is characterized by inflammation of synovial lining and articular shoulder capsule, leading to pain, stiffness and restricted mobility ${ }^{[3]}$. The pathology affects the glenohumeral capsular tissue and is particularly localised to the coracohumeral ligament in the rotator interval. Analysis of this tissue has shown inflammatory changes, fibrosis and proliferative myelofibrosis. This process may be cytokine mediated ${ }^{[4]}$.

Many treatments are suggested by orthopaedic professionals to the patients of Frozen Shoulder Syndrome (FSS). Some suggest different nonoperative treatments like physical therapy, exercises, articular stretching and pulley therapy and some suggest physical therapy with medication. Intensive physical therapies including passive stretching and manual mobilization have shown average results. Similarly, low oral corticosteroid has potential hyperglycemic effect. But, the combination of physical therapy and medication have shown better results ${ }^{[5]}$.

We evaluated the role of intraarticular corticosteroid injection because it decreases inflammation which leads to reduction in capsular fibrosis. This allows enhancement of joint motion and reduces the functional recovery time ${ }^{[6]}$.

\section{Materials \& Methods}

Study Design:

It is an observational study with a sample size of 30 .

\section{Dr. Anil Juyal}

Professor Orthopaedics,

Professor Orthopaedics

Himalayan Institute of Medical

Sciences, Dehradun,

Uttarakhand, India 
Consecutive reporting frozen shoulder patients in the OPD were recruited. All patients (>40 year) with $\mathrm{U} / \mathrm{L}$ or B/L frozen shoulder with duration of pain more than or equal to two months were included. Patients with previous history of trauma or surgery to the concerned shoulder, Systemic disorders like Rheumatoid Arthritis or any bleeding disorder, Any recent history of aspirin or aspirin like drug intake, history of diabetes mellitus, Platelet count $<1.5 \mathrm{lakh} / \mathrm{cu} \mathrm{mm}$ were excluded from the study.

The Study Tool used in this study were case reporting form to generate data, Constant shoulder score (CSS) (7), Visual Analogue Scale (VAS) (8) for scoring and Steroid (Methyl
Prednisolone) for intraarticular administration.

\section{Results}

The study comprised of 32 subjects with primary diagnosis of Frozen shoulder (Adhesive Capsulitis and Periarthritis). All the recruited subjects were given Intra-articular Corticosteroid injection after manipulation under General Anaesthesia. Outcome of the intervention were assessed at three different time frames i.e. 2, 6 and 12 weeks. Pain was assessed by VAS score (Visual analogue Scale) and Shoulder movements were assessed by CSS (Constant Shoulder Score).

Table 1: Distribution of cases according to VAS Score at different time interval $(n=32)$

\begin{tabular}{|c|c|c|c|c|}
\hline \multicolumn{5}{|c|}{ Number of cases } \\
\hline & Pre intervention & \multicolumn{3}{|c|}{ Post intervention } \\
\hline VAS Score & 0 Week & 2 Weeks & 6 Weeks & 12 Weeks \\
\hline $0-3$ & $1(3.13 \%)$ & $9(28.13 \%)$ & $27(84.38 \%)$ & $32(100 \%)$ \\
\hline $4-6$ & $10(31.25 \%)$ & $22(68.75 \%)$ & $5(15.63 \%)$ & 0 \\
\hline $7-10$ & $21(65.63 \%)$ & $1(3.13 \%)$ & 0 & 0 \\
\hline Total & 32 & 32 & 32 & 32 \\
\hline
\end{tabular}

Grading: 0-3 Mild 4-6 Moderate 7-10 Severe

Before intervention, $21(65.63 \%)$ out of 32 cases had VAS score between 7-10. After intervention it was seen that VAS score improved and 22 cases $(68.75 \%)$ had $4-6$ score at 2 weeks. At 6 weeks, 27 cases $(84.38 \%)$ and at 12 weeks all the 32 cases had VAS score between 0-3.

Table 2: Statistical evaluation of VAS score (pre and post injection)

\begin{tabular}{|c|c|c|c|}
\hline \multirow{2}{*}{ VAS score } & \multicolumn{2}{|c|}{ Number of cases } & \multirow{2}{*}{ Statistical evaluation } \\
\cline { 2 - 3 } & Pre intervention & Post intervention (12 weeks) & \multirow{2}{*}{$\chi^{2}=60.12, p<0.005$} \\
\hline $0-3$ & $1(3.12 \%)$ & $32(100 \%)$ & \\
\hline $4-6$ & $10(31.25 \%)$ & $0(0.0 \%)$ & $0(0.0 \%)$ \\
\hline
\end{tabular}

The difference in the VAS score in pre and post intervention phase was found to be highly significant statistically $\left(\chi^{2}=60.12, p<0.005\right)$.

Table 3: Distribution of cases according to Constant Shoulder Score at different time interval $(n=32)$

\begin{tabular}{|c|c|c|c|c|}
\hline \multicolumn{5}{|c|}{ Number of cases } \\
\hline \multirow{2}{*}{ Constant Shoulder Score } & \multirow{2}{*}{ Pre intervention } & \multicolumn{3}{|c|}{ Post intervention } \\
\cline { 3 - 5 } & & 2 week & 6 week & 12 week \\
\hline$<11$ & $0(0.0 \%)$ & $0(0.0 \%)$ & $0(0.0 \%)$ & $0(0.0 \%)$ \\
\hline $11-20$ & $26(81.25 \%)$ & $0(0.0 \%)$ & $0(0.0 \%)$ & $0(0.0 \%)$ \\
\hline $21-30$ & $5(15.62 \%)$ & $0(0.0 \%)$ & $0(0.0 \%)$ & $0(0.0 \%)$ \\
\hline$>30$ & $1(3.12 \%)$ & $32(100 \%)$ & $32(100 \%)$ & $32(100 \%)$ \\
\hline
\end{tabular}

Before intervention, in majority of the cases i.e $26(81.25 \% \%)$ CSS was found to be between 11-20. However in subsequent follow ups at 2 weeks, 6 weeks and 12 weeks, CSS of all the subjects i.e $32(100 \%)$

Table 4: Statistical evaluation of Constant Shoulder Score (pre and post injection)

\begin{tabular}{|c|c|c|c|}
\hline \multirow{2}{*}{ Constant Shoulder Score } & \multicolumn{2}{|c|}{ Number of cases } & Statistical evaluation \\
\hline & Pre intervention & Post intervention (12 week) & \multirow{5}{*}{$\chi^{2}=60.12, p<0.005$} \\
\hline$<11$ & $0(0.0 \%)$ & $0(0.0 \%)$ & \\
\hline $11-20$ & $26(81.25 \%)$ & $0(0.0 \%)$ & \\
\hline $21-30$ & $5(15.62 \%)$ & $0(0.0 \%)$ & \\
\hline$>30$ & $1(3.12 \%)$ & $32(100 \%)$ & \\
\hline
\end{tabular}

The difference in the CSS score in pre and post intervention phase was found to be highly significant $\left(\chi^{2}=60.12, p<0.005\right)$.

\section{Discussion}

The mean age group of the patients in this study is $53.15 \pm$ 10.62 years. Age ranged between 20 and 80 years. Maximum numbers of patients were seen in the age group of 51- 60 years. A female preponderance was observed in this study. In the present study $22(68.75 \%)$ subjects were female and 10 $(31.25 \%)$ subjects were males, similar observation was seen by Wang JP el at in his study in which out of 63 patients 23 were male and 40 were female ${ }^{[9]}$. In our study most of the cases were housewives, being $20(62.5 \%)$ cases followed by farmers being $8(25 \%)$ which may due to the fact that housewives has limited outdoor physical activities and more of sedentary lifestyle. As the incidence recorded all females were found to be performing static household activities.

Left side frozen shoulder was encountered more frequently than right side in this study. $17(53.12 \%)$ patient had frozen shoulder of left side (non dominant) while $13(40.62 \%)$ had right side (dominant) and $2(6.25 \%)$ had bilateral side. Similar 
observations were made by Siraj M et al. in their study where 113 subjects were studied out of which 47 had involvement of right shoulder (dominant) and 66 had involvement of left shoulder (non dominant) ${ }^{[10]}$.

We evaluated pain and Range of Motion till 12 weeks of intervention and the results were found to be statistically significant with $p<0.05$. There were no complications during or after the intervention like shoulder stiffness, infection, reflex sympathetic Dystrophy, post injection flare facial flushing, neurovascular damage etc.

MN Rudra et al. conducted a study in which he took two groups, one with Arthroscopic release of capsule and another with intra-articular steroid injection and he found that there is significant improvement in VAS and CSS score with a $P<0.005$ in surgical release group although in our study we have single group of patients but we obtained statistically significant VAS and CSS score with $P<0.05$ after mobilization under short GA followed by giving intraarticular steroid injection (11). Aslani $\mathrm{H}$ et al. conducted a study in which he took two groups, one with Intra-articular steroid and another with Intra-articular Platelet rich plasma with mobilization under GA. He found a significant improvement by using a platelet rich plasma instead of steroid but in our study we obtained significant improvement in pain and shoulder movement after mobilization under short GA followed by giving intra-articular steroid injection ${ }^{[12]}$.

Shah Nicholas conducted a study in which 40 patients of frozen shoulder were mobilized under general anaesthesia followed by 3 doses of Steroid at regular interval. Pain and range of motion was evaluated by VAS and CSS score at a regular interval of 6 weeks and he found that there is significant improvement with a $P<0.05$ in VAS and CSS score which was similar to our study ${ }^{[13]}$.

\section{Limitation of our study}

Sample size is small.

No comparison group.

\section{Conclusion}

In our study of 32 cases of frozen shoulder, all are mobilized under GA followed by Intra-articular steroid injection. All the patients were evaluated at regular interval for 12 weeks by VAS and CSS score. On the basis of this study we can conclude that:-

- There were significant improvement in pain and functional score after intervention.

- Intra-articular steroid injection with manipulation is safe and effective modality of the treatment for frozen shoulder.

- It is a cost effective procedure which can be performed on OPD basis.

\section{References}

1. Diercks RL, Stevens M. Gentle thawing of the frozen shoulder: a prospective study of supervised neglect versus intensive physical therapy in seventy-seven patients with frozen shoulder syndrome followed up for two years. J Shoulder Elbow Surg. 2004; 13(5):499-502.

2. Marx RG, Robert W, Kainter K, Wickiewicz TL. Intraarticular corticosteroid injection for the treatment of idiopathic adhesive capsulitis of the shoulder. HSSJ. 2007; 3:202-207.

3. Frontra G. Essentials of Physical Medicine and Rehabilitation. 2nd ed. Philadelphia: Saunders, Elsevier, Adhesive capsulitis, 2008, 49-54.
4. Dias R, Cutts S, Massoud S. Frozen shoulder. BMJ. 2005; 331:1453-6.

5. Tveita EK, Sandvik L, Ekeberg OM, Juel NG, BautzHolter E. Factor structure of the Shoulder Pain and Disability Index in patients with adhesive capsulitis. BMC Musculoskelet Disord. 2008; 9:103.

6. Bunker T, Reilly J, Baird K, Hamblen D. Expression of growth factors, cytokines and matrix metalloproteinases in frozen shoulder. J Bone Joint Surg [Br]. 2000; 82(B):768-73.

7. Constant CR, Murley AH. A clinical method of functional assessment of the shoulder. Clin Orthop Relat Res. 1987; 214:160-4.

8. Gould D. Visual analog score (VAS). J Clin Nursing. 2001; 10:697-706.

9. Wang JP, Huang TF, Ma HL, Hung SC, Chen TH. Manipulation under anaesthesia for frozen shoulder in patients with and without non-insulin dependent diabetes mellitus. Int Orthop. 2010; 34(8):1227-32.

10. Siraj M, Anwar W, Iqbal MJ, Rahman N. Effectiveness of intra-articular corticosteroid injection in the treatment of idiopathic frozen shoulder. J Surg Pak. 2012; 17:5762.

11. Mukherjee RN, Pandey RM, Nag HL, Mittal R. Frozen shoulder - A prospective randomized clinical trial. World J Orthop. 2017; 8(5):394-99.

12. Aslani H, Nourbakhsh ST, Zafarani Z, Bani MA, Ebrahim M. Platelet rich plasma for frozen shoulder: A case report. Arch Bone Jt Surg. 2016; 4(1):90-93.

13. Shah N. Shoulder adhesive capsulitis: systematic review of randomized trials using multiple corticosteroid injections. Br. J. Gen Prac. 2007; 57(54):27-35. 